\title{
Knowledge of Breast Self-Examination, Breast and Cervical Cancer Screening among Indian Women
}

\author{
Olabode Omotoso ${ }^{1 *}$, Sucheta Malakar ${ }^{2}$, Nabanita Chutia ${ }^{3}$, Ghadier Matariek ${ }^{4}$, \\ Ghada Mahmoud Abdel-Rafee ${ }^{5}$, Elizabeth Omotoso ${ }^{6}$ \\ ${ }^{1}$ Department of Biochemistry, University of Ibadan, Ibadan, Nigeria. \\ ${ }^{2}$ Vijaygarh Jyotish Ray College, Calcutta University, Kolkata, India. \\ ${ }^{3}$ Centre for Biotechnology and Bioinformatics, Dibrugarh University, India. \\ ${ }^{4}$ Faculty of Pharmacy, Mansoura University, Mansoura, Egypt. \\ ${ }^{5}$ Mansoura Health Insurance Organization, Mansoura, Egypt. \\ ${ }^{6}$ Faculty of Education, University of Ibadan, Ibadan, Nigeria.
}

Received 27 February 2021; Revised 20 July 2021; Accepted 10 October 2021; Published 01 December 2021

\begin{abstract}
The burden of breast and cervical cancer in terms of incidence and mortality in low- and mid-income countries is increasing daily due to late diagnosis, unhealthy lifestyle choices, late presentation, and poor attitude to screening. Early detection increases the chances of survival. This present study assessed Assam women's breast and cervical cancer screening (CCS) awareness, the practice of breast self-examination (BSE), and uptake of human papillomavirus vaccines. An internet-based cross-sectional questionnaire was utilized to obtain 251 consenting respondents. The mean age of respondents was $27.8 \pm 6.91$. Only $205(81.7 \%)$ and $110(43.8 \%)$ respondents have satisfactory levels of BCS, and CCS knowledge, respectively. While only $76(30.3 \%)$ respondents had satisfactory BSE practice. Age range (26 - 32 years), tertiary education, and being single were demographic characteristics that influenced knowledge and practice. Though the respondents had a satisfactory knowledge level, screening uptake among respondents is very poor as only $32(12.7 \%)$ had ever been screened and only $19(7.6 \%)$ ever been vaccinated. The major reasons for poor screening uptake were "no symptoms" and "not aware of screening location". Most respondents $(227,90.4 \%)$ are willing to go for screening if well oriented. This presents an opportunity to enhance awareness about screening and vaccination among Assam women. The media/internet and health practitioners can be leveraged to promote the uptake and utilization of screening services and BSE practice.
\end{abstract}

Keywords: Breast Self-examination; Cancer Screening; Early Detection; HPV; Awareness.

\section{Introduction}

Breast and cervical cancer are major public health challenges. Globally, an estimated 19.3 million new cancer cases and about 10 million cancer deaths were reported in 2020 out of which 1,324,413 new cancer cases with 851,678 deaths were reported in India [1]. 178,361 (26.3\%) breast cancer cases and 123,907 (18.3\%) cervical cancer cases were the most prevalent of the new 678,383 cancer cases reported in Indian women in 2020 [1]. Hence, breast and cervical cancer are the leading cause of cancer burden in Indian women.

* Corresponding author: olabodeomotoso@gmail.com

\section{doi) http://dx.doi.org/10.28991/SciMedJ-2021-0304-5}

$>$ This is an open access article under the CC-BY license (https://creativecommons.org/licenses/by/4.0/).

(C) Authors retain all copyrights. 
The burden of breast and cervical cancer in terms of incidence and mortality in low- and mid-income countries is increasing daily due to late diagnosis, unhealthy lifestyle choices, poor attitude, and late presentation for screening [2]. Prevention is the best strategy for effective cancer management. Almost all cancer cases especially breast and cervical cancer are highly preventable and have high chances of survival if detected early [3]. Promotion of national cancer screening strategies to ensure early detection has contributed to better outcomes in developed countries [3]. Sadly, late presentation for screening or diagnosis is a common limitation to optimal cancer care in low- and mid-income countries [2].

The human papillomavirus (HPV) has been found culpable in most cervical cancer cases [4]. The development and uptake of HPV vaccines (bivalent Cervarix and quadrivalent Gardasil) have contributed immensely to the decline in cervical cancer cases in developed nations [3]. It is crucial to identify common factors that increase and reduce the risk of breast cancer in women (Figure 1). Most breast cancer cases are self-reported when women begin to experience signs and symptoms suggesting breast abnormalities and discomfort [5]. Hence, detection of breast cancer in early stages via the non-invasive, simple, and no- or low-cost breast self-examination (BSE) can be a promising strategy in reducing the breast cancer burden. Despite the importance of BSE and its cost-effectiveness, earlier reports [5-7] have shown very poor knowledge and uptake among women. Earlier studies [2, 3] have identified a lack of awareness, attitude, or knowledge as contributory factors to the low uptake or underutilization of cancer preventive strategies. The effort of health institutions and health-based Non-Governmental Organizations (NGOs) in providing screening services (clinical breast examination, mammography, Pap smear test, and visual inspection with acetic acid) has contributed immensely to the uptake of opportunistic breast and cervical cancer screening among women in developing nations.



Figure 1. Common factors that increase (left) and reduces (right) the risk of breast cancer

Presentation for screening facilitates early detection. Uptake of breast cancer screening (BCS) and cervical cancer screening (CCS) services is hinged on awareness and the right attitude towards screening [2]. Several studies have been carried out to assess the awareness, knowledge, practice, and attitude of Indian women about BCS, BSE, and CCS [5, 6, 8-11]. An earlier study [6] assessed the knowledge of 314 women attending the Prathipadu Health Centre, a rural area in South India, with a reported $30.9 \%$ awareness about BSE with only $22.6 \%$ women practicing it. A study involving 356 female Information Technology (IT) professionals in India's Silicon Valley [5], identified that BSE knowledge (mean score $18.17 \pm 2.90$ ) and practice (mean score $19.11 \pm 5.08$ ) were extremely correlated. Other studies $[13,14]$ involving Indian women have also reported poor BSE knowledge and/or practice. In another study [12] involving 320 Sikkimese nursing staff, the majority (79.1\%) were aware of CCS, meanwhile, only $11.9 \%$ had ever undergone CCS. Poor CCS uptake was likewise observed among female healthcare workers in the United Arab Emirates [15] and Turkish women [16].

Breast and cervical cancers are not spontaneous but develop with age and time [2], hence, the present study aims to assess the knowledge of Assam women about BCS, CCS, and BSE practice. The present study focused more on young 
women, the reason being older women have more tendencies to uptake breast or cervical cancer screening. Having possibly gone through higher education, pregnancies, breastfeeding and parenting, which might have increased their chances for opportunistic screening and knowledge about BSE, BCS, and CCS [5, 17]. We likewise identified respondents' sources of information about the subject and reasons for not utilizing breast and cervical cancer screening services. Findings from this study would help to understand how best to curb the menace of breast and cervical cancer among the study population.

\section{Material and Methods}

\subsection{Study Participants and Sampling}

A slightly modified methodology [2] was utilized (Figure 2). This cross-sectional study aimed at understanding and promoting strategies to reduce the burden of breast and cervical cancers in Assam was carried out between October and November 2020. Using a convenient sampling method, 251 young women who consented and are above 18 years were recruited. Men, non-consenting women, and women below 18 years and above 45 years of age were excluded from the study. The assessment was based on questions addressing respondents' knowledge and uptake of BCS and CCS, HPV vaccination and practice of BSE.



Figure 2. Flowchart illustrating the research methodology

\subsection{Questionnaire Design}

The use of a paper-based questionnaire was not feasible due to the global coronavirus pandemic; we thereby restricted our study to women with internet access. The administered questionnaire was designed using Google forms (Alphabet Inc., California, USA) and pre-validated by two independent reviewers. The questionnaire was pre-tested on 20 respondents who were not included in the analysis. The questionnaire consists of 4 major parts:

- Sociodemographics;

- Knowledge of BCS and CCS;

- Uptake of the HPV vaccine;

- Practice of BSE.

We also assessed respondents' major sources of information about cancer screening, reasons for not yet being screened (for those who are not) and willingness to be screened if well-educated about cancer screening. The questionnaire is available in the Appendix I.

\subsection{Data Analysis}

Responses were imported from Google form as a Microsoft Excel spreadsheet. All data obtained were then analyzed using the Statistical Package for the Social Sciences software, v.20. Descriptive statistics were used to summarize respondents' sociodemographic information. Respondents' knowledge of breast and cervical cancer screening and practice of BSE was assessed using a numbered scoring pattern ( 1 for a correct response and 0 for an 
incorrect response). The dependent variables were computed and then binned into an equal percentile (50\%) based on mean scores to categorize the knowledge level. Respondents with numeric scores greater than the mean scores were classified as good or satisfactory knowledge level, and vice-versa. Preliminary analyses were performed to ensure no violation of the assumptions of normality, linearity, and homoscedasticity. Correlation analysis was performed to test for relationships between outcome variables. Independent sample t-test and one-way analysis of variance were used to test for mean differences in the knowledge score across the independent variables with two and three groups, respectively. Significant relationships $(\mathrm{P}<0.05)$ were subjected to further significant tests.

\section{Results}

\subsection{Participant's Demographics}

A total of 251 consenting women were included in this present study. The mean age of respondents is $27.8 \pm 6.91$. Most respondents ( $90 \%, \mathrm{n}=226 / 251)$ were single (never married). Similarly, most of the respondents have at least a University degree $(80.1 \%, \mathrm{n}=201 / 251)$ compared to other women with a low educational level $(19.9 \%)($ Table 1$)$.

Table 1. Demographics of respondents used in this study $(n=251)$

\begin{tabular}{ccc}
\hline Variable & & Number of respondents (\%) \\
\hline \multirow{2}{*}{ Age (years) } & $18-23$ & $89(35.5 \%)$ \\
& $24-32$ & $84(33.5)$ \\
& $33-45$ & $78(31 \%)$ \\
\hline \multirow{2}{*}{ Education } & Primary/Secondary & $50(19.9 \%)$ \\
& Bachelors & $135(53.8 \%)$ \\
& Postgraduate & $66(26.3 \%)$ \\
\hline \multirow{2}{*}{ Marital status } & Single & $226(90 \%)$ \\
& Ever married & $25(10 \%)$ \\
\hline
\end{tabular}

* Results are presented as frequencies and corresponding percentages in a bracket

\subsection{Knowledge of BCS, CCS and BSE Practice among Respondents}

Only $205(81.7 \%)$ and $110(43.8 \%)$ respondents have satisfactory levels of BCS, and CCS knowledge, respectively. While only $76(30.3 \%)$ of the respondents had satisfactory BSE practice. BCS and CCS knowledge was satisfactory across all age groups, while young women in the age range (24-32 years) have satisfactory levels of BSE practice compared to other age groups. Respondents who had education up to the tertiary level had satisfactory knowledge of BCS (82\%), CCS (72.7\%), and BSE practice (73.7\%) compared to young women with a lower educational level. Based on marital status, respondents who are single (never married) had satisfactory BCS knowledge (82.3\%), CCS knowledge (42\%), and BSE practice (29.2\%) compared to those that are married (Table 2).

Table 2. Relationship between sociodemographic data and outcome variables

\begin{tabular}{|c|c|c|c|c|}
\hline Variable & & Unsatisfactory $(<\mathbf{5 0 \%})$ & Satisfactory $(\geq \mathbf{5 0} \%)$ & Total \\
\hline \multicolumn{5}{|c|}{ Sociodemographic data and BCS knowledge } \\
\hline \multirow{3}{*}{ Age (years) } & $18-23$ & $16(18 \%)$ & $73(82 \%)$ & 89 \\
\hline & $24-32$ & $15(17.9 \%)$ & $69(82.1 \%)$ & 84 \\
\hline & $33-45$ & $15(19.2 \%)$ & $63(80.8 \%)$ & 78 \\
\hline \multirow{3}{*}{ Education } & Primary/Secondary & $13(26 \%)$ & $37(74 \%)$ & 50 \\
\hline & Bachelors & $24(17.8 \%)$ & $111(82.2 \%)$ & 135 \\
\hline & Postgraduate & $9(13.6 \%)$ & $57(86.4 \%)$ & 66 \\
\hline \multirow{2}{*}{ Marital status } & Single & $40(17.7 \%)$ & $186(82.3 \%)$ & 226 \\
\hline & Ever married & $6(24 \%)$ & $19(76 \%)$ & 25 \\
\hline \multicolumn{5}{|c|}{ Sociodemographic data and CCS knowledge } \\
\hline \multirow{3}{*}{ Age (years) } & $18-23$ & $49(55.1 \%)$ & $40(44.9 \%)$ & 89 \\
\hline & $24-32$ & $50(59.5 \%)$ & $34(40.5 \%)$ & 84 \\
\hline & $33-45$ & $42(53.8 \%)$ & $36(46.2 \%)$ & 78 \\
\hline \multirow{3}{*}{ Education } & Primary/Secondary & $20(40 \%)$ & $30(60 \%)$ & 50 \\
\hline & Bachelors & $88(65.2 \%)$ & $47(34.8 \%)$ & 135 \\
\hline & Postgraduate & $33(50 \%)$ & $33(50 \%)$ & 66 \\
\hline
\end{tabular}




\begin{tabular}{ccccc}
\hline \multirow{2}{*}{ Marital status } & Single & $131(58 \%)$ & $95(42 \%)$ & 226 \\
& Ever married & $10(40 \%)$ & $15(60 \%)$ & 25 \\
\hline \multirow{3}{*}{ Age (years) } & Sociodemographic data and BSE practice & & 89 \\
& $18-23$ & $64(71.9 \%)$ & $25(28.1 \%)$ & 84 \\
& $24-32$ & $53(63.1 \%)$ & $31(36.9 \%)$ & 78 \\
\hline \multirow{2}{*}{ Education } & $33-45$ & $58(74.4 \%)$ & $20(25.6 \%)$ & 50 \\
& Primary/Secondary & $30(60 \%)$ & $20(40 \%)$ & 135 \\
& Bachelors & $100(74.1 \%)$ & $21(31.8 \%)$ & 66 \\
\hline \multirow{2}{*}{ Marital status } & Postgraduate & $45(68.2 \%)$ & $66(29.2 \%)$ & 226 \\
& Single & $160(70.8 \%)$ & $10(40 \%)$ & 25 \\
\hline
\end{tabular}

$107(42.6 \%)$ respondents identified the media/internet as their major source of information about breast and cervical cancer. Although most respondents (86.5\%) believe breast or cervical cancer is preventable and that early detection increases the chances of survival $(91.6 \%)$, some of the respondents $(33.9 \%)$ opine that BCS and CCS are only meant for those feeling symptoms. Out of the $32(12.7 \%)$ respondents who have at a time observed abnormalities or lumps, most of them consulted healthcare giver (44\%) and others did nothing while the symptoms disappeared with time $(37 \%)$.

Out of the $214(85.3 \%)$ respondents who have ever heard of cervical cancer, only 107 (42.6\%) are aware of CCS tests and 73 (29.1\%) know where to be screened. Most respondents ( $87.3 \%$ ) have never undergone CCS, with most identifying no symptoms $(69.7 \%)$, and not being aware of where to be screened $(17.9 \%)$ as major reasons for not being screened. Only $52(20.7 \%)$ respondents have heard of the HPV vaccines. Likewise, only 19 (7.6\%) respondents have ever been vaccinated with the HPV vaccine. About half of the respondents (58.6\%) are aware of BSE; however, only a very few $(24.7 \%)$ can perform BSE effectively. 158 (62.9\%) respondents identified not being taught as the major reason for not performing BSE. The majority of participants (90.4\%) are willing to be screened if properly oriented (Supplementary S1).

\subsection{Influence of Sociodemographic Factors on BSE Practice, BCS and CCS Knowledge}

The influence of educational qualification on CCS and BCS knowledge was explored. Levene's test significance value of 0.06 depicts non-violation of the homogeneity of variance assumption. There was a statistically significant difference at the $\mathrm{P}<0.05$ level in CCS knowledge for the three educational statuses $[\mathrm{F}(2,248)=5.569, \mathrm{P}=0.026]$. The Post-hoc comparisons using the Tukey HSD multiple comparison test indicated that the mean score was significantly different for respondents who had primary/secondary education and those with a Bachelor's degree; the effect size (0.043), calculated using eta squared depicts a medium effect size.

There was a statistically significant difference at the $\mathrm{P}<0.05$ level in BCS knowledge for the three educational statuses $[\mathrm{F}(2,248)=5.591, \mathrm{P}=0.04]$. The Post-hoc comparisons using the Tukey HSD multiple comparison test indicated that the mean score was significantly different for respondents across the three educational statuses; the effect size (0.043) depicts a medium effect size. As shown in Table 2, a satisfactory level of knowledge of CCS differs across the educational status. Meanwhile, the mean difference in BSE practice was not significant across the three educational statuses.

The relationship between knowledge of BCS and practice of BSE was investigated using Pearson product-moment correlation coefficient at 0.01 significance level. There was a small, positive correlation between the two variables $[\mathrm{r}=0.208, \mathrm{~N}=251, \mathrm{P}=0.001]$, with a good knowledge of breast cancer screening associated with a higher level of BSE practice. The coefficient of determination (4\%) depicts that there is not much overlap between knowledge of BCS and respondents' practice of BSE.

The relationship between knowledge of BCS and CCS was likewise investigated using Pearson product-moment correlation coefficient at 0.01 significance level. There was also a low, positive correlation between the two variables $[\mathrm{r}=0.312, \mathrm{~N}=251, \mathrm{P}=0.001]$, with a high knowledge level of BCS associated with satisfactory CCS knowledge. The coefficient of determination depicts that knowledge of BCS helps to explain nearly $10 \%$ of the variance in respondents' score on CCS.

An independent-samples t-test was conducted to compare the BCS, CCS knowledge, and BSE practice scores for the respondents based on marital status. For BCS knowledge, there was no significant difference in scores for single young women $(\mathrm{M}=2.77, \mathrm{SD}=0.72)$ and those that are married $[\mathrm{M}=2.76, \mathrm{SD}=0.83$; $\mathrm{t}(249)=0.064, \mathrm{P}=0.49]$. For $\mathrm{CCS}$ knowledge, there was also no significant difference in scores for single young women $(\mathrm{M}=1.42, \mathrm{SD}=0.5)$ and those 
that are married $[\mathrm{M}=1.6, \mathrm{SD}=0.5 ; \mathrm{t}(249)=0.721, \mathrm{P}=0.086]$. Likewise, there was no significant difference in BSE practice scores for single young women $(\mathrm{M}=1.29, \mathrm{SD}=0.456)$ and those that are married $[\mathrm{M}=1.4, \mathrm{SD}=0.5 ; \mathrm{t}(249)=$ $0.113, \mathrm{P}=0.267]$.

\section{Discussion}

To the best of our knowledge, this is the first study focusing on understanding young women's knowledge and screening of the two most dreaded cancers in women and BSE practice in Assam, India. The incidence and mortality due to breast and cervical cancer have posed a dire threat to global public health and the total wellbeing of the patients. When detected early, the chances of survival and better disease outcome are relatively high [18]. Developed nations have been able to leverage the promotion and uptake of screening services in curtailing the menace of cancer burden $[18,19]$. However, it is saddening that most breast and cervical cancer cases are presented late in developing nations.

The main highlight of this present study is that only $205(81.7 \%), 110(43.8 \%)$, and $76(30.3 \%)$ respondents have satisfactory levels of BCS, CCS knowledge, and BSE practice, respectively. The media/internet (42.6\%) and health practitioners $(16.7 \%)$ are the respondents' main sources of information. Earlier studies [9, 11] likewise identified family/friends, print and electronic media, and health practitioners as major sources of information. Although most respondents $(91.6 \%)$ believe early detection promotes survival, only $12(4.8 \%)$ and $32(12.7 \%)$ have ever been screened or examined for breast and cervical cancer, respectively. The respondents identified no symptoms, not being aware of screening centers, and screening cost consideration as major reasons for not being screened.

The poor BSE practice observed in our study corroborates an earlier study [6] in South India where only $31 \%$ are aware of BSE and only $22.6 \%$ practice BSE and a study [7] of 206 women in Andhra Pradesh, India where $16.5 \%$ are aware of BSE but only very few $(2.4 \%)$ are practicing BSE. A recent similar study conducted among female IT professionals in Silicon Valley, India [5] reported that although the majority $(62.4 \%)$ of the respondents are knowledgeable about BSE, about $68 \%$ had a poor attitude and only 134 (37.6\%) had a good practice. Despite the relative ease and no cost implication for BSE [17, 20], sadly, most women fail to leverage this important age-long technique. The major reasons for not performing BSE in our study include not being taught, forgetfulness, fear of discovering abnormalities, and being too busy. In contrast to our study, BSE practice was found to be significantly associated with age and educational status in an earlier report [6].

Our study showed a slight improvement over a study [9] of 1140 tribal women in South India where none of the respondents had undergone CCS and that of 407 rural Indian women [11] where most respondents (98.5\%) had poor knowledge regarding CCS. Likewise, among 320 Nursing staff in Sikkim, India [12], although most respondents (79.1 $\%)$ were aware of CCS, only very few (11.9\%) had ever undergone screening. About half of the 1006 respondents in a bi-national study [2] in Africa involving Egyptian and Nigerian women had good knowledge (49.5\%) of CCS, meanwhile, only $41 \%$ knew where to be screened and very few $(11 \%)$ had ever undergone CCS. Likewise, in a study of 1033 women residing in a low-income urban community in India [10], only very few (7.1\%) had ever undergone CCS. Also, despite good CCS knowledge, only 24 (8.3\%) of 290 community healthcare workers in Uttar Pradesh, India have undergone CCS [21]. Major reasons given were "will only go when there is a problem or complaint" (35.7 $\%$ ), fear of pain $(24.6 \%$ ), and no knowledge of where CCS can be done (23.8\%) [21]. Long distances to where CCS services can be accessed $(86.5 \%)$, unavailability of CCS services at local healthcare centers $(84 \%)$, and religious constraint (39.7\%) were the major barriers to CCS uptake among Zimbabwean women [22].

Only $52(20.7 \%)$ respondents have heard of the HPV vaccines. Likewise, only 19 (7.6\%) respondents have ever been vaccinated with the HPV vaccine. The poor vaccine uptake was also found among 1033 Indian women living in a low-income urban community [10] where none of them has ever received the HPV vaccines. This corroborates an earlier study where only $2.2 \%$ of the 1006 Egyptian and Nigerian women had ever been vaccinated with the HPV vaccines [2]. The high cost of the HPV vaccines could have been a major factor for not receiving the vaccines [10].

The major limitations in our study are the use of an internet-based questionnaire and the sample size. We had to result using an internet-based questionnaire due to the current pandemic. This has limited our study to those who had internet access, which might have in a way contributed to the identification of the internet as respondents' major source of information. Hence, we cannot generalize our result to the whole Indian population. Despite this, our study in light of earlier reports has been able to present more evidence on the need for the Government, organizations, policymakers and individuals to harness all resources to put an end to needless and preventable death due to breast and cervical cancer.

\section{Conclusion}

Breast and cervical cancer are the leading cause of cancer death in Indian women. There is an increased chance of survival when breast and cervical cancer are detected early. Our study identified poor screening, BSE practice, and vaccination uptake among the respondents. We have also been able to identify major barriers to the utilization of 
breast and cancer screening services. Breast and cervical cancer screening services and HPV vaccines should be subsidized, especially for high-risk women to increase accessibility and affordability. The respondents' willingness to go for screening $(90.4 \%)$ presents an opportunity to enhance awareness about screening and vaccination among women. It is not only important to provide health and screening facilities, but women must also be educated on why it is important to be screened and where to be screened. The media/internet and health practitioners can be leveraged on as they have been reported in our study and several other studies as major sources of breast and cervical cancer information. Future studies should focus on assessing the awareness and uptake of BSE, breast and cervical cancer screenings, and HPV vaccination in older and other women with no access to the internet.

\section{Declarations}

\subsection{Author Contributions}

Conceptualization, O.O.; methodology, S.M., N.C., G.M.; data analysis, O.O.; resources, S.M., G.A. and N.C.; writing — original draft preparation, O.O.; writing—review and editing, S.M., N.C., G.M., and G.A. All authors have read and agreed to the published version of the manuscript.

\subsection{Funding}

The authors received no financial support for the research, authorship, and/or publication of this article.

\subsection{Acknowledgements}

We appreciate all respondents for their time and consent to be part of this study.

\subsection{Ethical Approval}

A short introduction clearly stating the aim of the study, voluntary participation, anonymity and the safety of all data collected was presented to all respondents before completing the survey. Being a low-risk study, anonymity upheld and non-inclusion of participants under 18 years, informed consent was obtained. The email of the principal investigator was supplied for any clarification or withdrawal of participation and the investigators adhered to maximal ethical compliance in line with the World Medical Association Declaration of Helsinki Ethical principles.

\subsection{Data Availability Statement}

The data presented in this study are available in article and supplementary material.

\subsection{Conflict of Interest}

The authors declare that they have no known competing financial interests or personal relationships that could have appeared to influence the work reported in this paper.

\section{References}

[1] Global Cancer Observatory. Available online: https://gco.iarc.fr/ (accessed on March 2021).

[2] Omotoso, O. E., Matariek, G., Omotoso, E. F., Matareek, A., Abdul-Rafee, G. M., Malakar, S., \& Chutia, N. (2021). Practice of Breast Self-Examination and Knowledge of Breast and Cervical Cancer Screening. SciMedicine Journal, 3(3), $219-229$. doi:10.28991/scimedj-2021-0303-3.

[3] Heena, H., Durrani, S., AlFayyad, I., Riaz, M., Tabasim, R., Parvez, G., \& Abu-Shaheen, A. (2019). Knowledge, Attitudes, and Practices towards Cervical Cancer and Screening amongst Female Healthcare Professionals: A Cross-Sectional Study. Journal of Oncology, 2019, 1-9. doi:10.1155/2019/5423130.

[4] WHO. Cervical cancer. WHO Africa. 2020. Available online: https://www.afro.who.int/health-topics/cervical-cancer (accessed on December 2020).

[5] Gore, C., Kalliguddi, S., \& Sharma, S. (2019). Knowledge, attitude, and practice of breast self-examination amongst female IT professionals in Silicon Valley of India. Journal of Family Medicine and Primary Care, 8(2), 568. doi:10.4103/jfmpc.jfmpc_315_18.

[6] Yerpude, P. N., \& Jogdand, K. S. (2013). Knowledge and practice of breast self-examination (BSE) among females in a rural area of South India. Natl J Community Med, 4(2), 329-332.

[7] Lakshmi A, Devi S, Borra S, Kommula V. (2014). Awareness and Practice of Breast Self-Examination among Women in South India. Int J Curr Microbiol Appl Sci. 3(1):391-4.

[8] Rani S. (2017). Assessment of Knowledge on Breast Self- Examination (BSE) among Women. Int J Adv Res. 5(9):200-7. doi:10.21474/IJAR01/5317 
[9] Ghosh, S., Mallya, S. D., Shetty, R. S., Pattanshetty, S. M., Pandey, D., Kabekkodu, S. P., ... Kamath, V. G. (2020). Knowledge, Attitude and Practices towards Cervical Cancer and its Screening among Women from Tribal Population: a Community-Based Study from Southern India. Journal of Racial and Ethnic Health Disparities, 8(1), 88-93. doi:10.1007/s40615-020-00760-4.

[10] Reichheld, A., Mukherjee, P. K., Rahman, S. M., David, K. V., \& Pricilla, R. A. (2020). Prevalence of Cervical Cancer Screening and Awareness among Women in an Urban Community in South India-A Cross Sectional Study. Annals of Global Health, 86(1). doi:10.5334/aogh.2735.

[11] Thovarayi, S., Noronha, J. A., \& Nayak, S. (2014). Knowledge of cervical cancer screening among rural Indian women: a cross sectional study. Journal of Nursing and Health Science, 3(3), 51-5.

[12] Rahman, H., \& Kar, S. (2015). Knowledge, attitudes and practice toward cervical cancer screening among Sikkimese nursing staff in India. Indian Journal of Medical and Paediatric Oncology, 36(2), 105-110. doi:10.4103/0971-5851.158840.

[13] Sachdeva, S., Mangalesh, S., \& Dudani, S. (2021). Knowledge, Attitude and Practices of Breast Self-Examination amongst Indian Women: A Pan-India Study. Asian Pacific Journal of Cancer Care, 6(2), 141-147. doi:10.31557/apjcc.2021.6.2.141147.

[14] Malik, R., Vera, N., Dayal, C., Choudhari, A., Mudaliar, J., Noovao Hill, A., ... Gunnarsson, R. (2020). Factors associated with breast cancer awareness and breast self-examination in Fiji and Kashmir India - a cross-sectional study. BMC Cancer, 20(1). doi:10.1186/s12885-020-07583-w.

[15] Soliman H, Zackaria SS, Mohammed HR. (2021). Breast, cervix and colorectal cancer attitude, knowledge and practice among eligible female health care workers in Umm AL Quwain primary health care centres. International Research Journal of Public and Environmental Health, 8(1): 48-56. doi:10.15739/irjpeh.21.007.

[16] Sevinc, N., Oral, B., \& Korkut, B. (2020). Assessment of Knowledge, Attitudes, and Behaviors of Turkish Women on Breast and Cervical Cancer in Karabük Province, Turkey. Kesmas: National Public Health Journal, 15(4): $211-218$. doi:10.21109/kesmas.v15i4.3865.

[17] Saran, C., Sandhiya, S. K., Yogeshkumar, S., \& Vignesh, T. (2020). Assessment of the Knowledge on Breast Self-examination among Women in Selected Tertiary Hospital at Kelambakkam, Kancheepuram District, Tamil Nadu, India. Medico Legal Update, 20(2), 183-187.

[18] WHO. Breast Cancer. Available online: https://www.who.int/news-room/fact-sheets/detail/breast-cancer (accessed on July 2021).

[19] Ewaid, S. H., Shanjar, A. M., \& Mahdi, R. H. (2018). Knowledge and practice of breast self-examination among sample of women in Shatra/Dhi-Qar/Iraq. Alexandria Journal of Medicine, 54(4), 315-317. doi:10.1016/j.ajme.2017.12.002.

[20] Parchuri, S. S., Palanki, S. D., Nirni, S. S., Atilli, S. S., Andra, V. V., \& Ch, V. M. (2019). Impact of education for breast self examination in rural Indian women on early detection: Results of POC study. Annals of Oncology, 30(5), v76-v77. doi:10.1093/annonc/mdz240.054

[21] Khargekar, N., Khanna, D., \& Budukh, A. (2019). Knowledge, attitude, and practice about cervical cancer and its screening among community healthcare workers of Varanasi district, Uttar Pradesh, India. Journal of Family Medicine and Primary Care, 8(5), 1715-1719. doi:10.4103/jfmpc.jfmpc_143_19.

[22] Nyamambi, E., Murendo, C., Sibanda, N., \& Mazinyane, S. (2020). Knowledge, attitudes and barriers of cervical cancer screening among women in Chegutu rural district of Zimbabwe. Cogent Social Sciences, 6(1), 1766784. doi:10.1080/23311886.2020.1766784. 


\section{Appendix I}

Practice of Breast Self-Examination and Knowledge of Breast and Cervical Cancer Screening.

Section A: Sociodemographic factors

1. Age in years:

2. Educational qualification:

No formal education or up to secondary school

Bachelor's degree

Postgraduate degree

3. Marital status

$\bigcirc$ Single or never married

Married

Section B: Cervical cancer screening and vaccination

1. Have you ever heard of cervical cancer?
$\bigcirc$ Yes
No
Maybe

2. Are you aware of screening tests for cervical cancer? (VIA or Pap smear test or other approved screening tests)?

$\bigcirc$ Yes

No

I am not sure

3. Do you know where to be screened?
Yes
$\bigcirc$ No
Maybe

4. Have you ever undergone cervical cancer screening (VIA or Pap smear test)?

$\bigcirc$ Yes

$\bigcirc \mathrm{No}$

I am not sure

5. Cervical screening can be done personally without a healthcare provider?

$\bigcirc$ Yes

$\bigcirc \mathrm{No}$

Maybe

6. Why have you not been screened?

$\bigcirc$ I have been screened

Not aware of where to be screened

$\bigcirc$ Lack of time

$\bigcirc$ Fear of positive result

Screening cost consideration

$\bigcirc$ No symptoms

$\bigcirc$ Other reasons 
7. Have you ever heard of HPV vaccines?
$\bigcirc$ Yes
$\bigcirc \mathrm{No}$
$\bigcirc$ Maybe

8. Have you received any of the HPV vaccines?
$\bigcirc$ Yes
$\bigcirc \mathrm{No}$
Maybe

9. Where do you get information about cervical or breast cancer?

$\bigcirc$ No knowledge of cervical or breast cancer

Health practitioners

$\bigcirc$ Media/internet

$\bigcirc$ Family/friends

School

Religious or social groups

$\bigcirc$ Others

10. If properly oriented about cervical or breast cancer, will you be willing to do the test (screening)?

$\bigcirc$ Yes

$\bigcirc$ No

Maybe

Section C - Breast cancer and Breast self-examination

1. Have you ever heard of breast cancer?

$\bigcirc$ Yes

$\bigcirc \mathrm{No}$

$\bigcirc$ Maybe

2. Positive diagnosis of breast cancer is a death sentence?

$\bigcirc$ Yes

$\bigcirc \mathrm{No}$

$\bigcirc$ Maybe

3. Is breast or cervical cancer preventable?
$\bigcirc$ Yes
$\bigcirc$ No

$\bigcirc$ Maybe

4. Cancer screening is only meant for those feeling symptoms.
$\bigcirc$ Yes
$\bigcirc$ No
Maybe

5. Early detection increases the chances of survival from breast cancer?
$\bigcirc$ Yes
$\bigcirc$ No
$\bigcirc$ Maybe 
6. Have you ever heard of breast self-examination (BSE)?
$\bigcirc$ Yes
$\bigcirc \mathrm{No}$
$\bigcirc$ Maybe

7. Have you been taught how to perform BSE?
$\bigcirc$ Yes
$\bigcirc$ No
$\bigcirc$ Maybe

8. Can you effectively perform BSE?
$\bigcirc$ Yes
$\bigcirc \mathrm{No}$
$\bigcirc$ Maybe

9. Why don't you perform BSE?

I have not been taught

I forget too often

I am too busy

$\bigcirc$ Fear of discovering lump or abnormalities

$\bigcirc$ Others

10. Have you ever been examined or screened for breast cancer? (Clinical Breast Examination or Mammogram or other approved screening tests)?

$\bigcirc$ Yes

$\bigcirc$ No

Maybe

11. How often should BSE be performed?

Daily

Weekly

Monthly

$\bigcirc$ Yearly

$\bigcirc$ Only when symptoms are felt

$\bigcirc$ Only during routine medical checkup

$\bigcirc$ I do not know

12. Have you ever observed lump or milky discharge or other abnormalities in your breast when not breastfeeding??

$\bigcirc$ Yes

$\bigcirc \mathrm{No}$

Maybe

13. If yes to above, what did you do?

$\bigcirc$ I used over-the-counter drugs

I consulted a healthcare provider

$\bigcirc$ I prayed over it

$\bigcirc$ I did nothing and it disappeared over time 